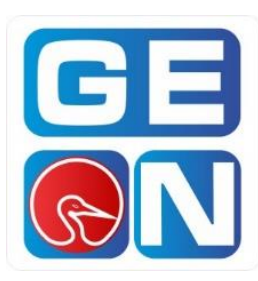

Revista GEON (Gestión, Organizaciones y Negocios.) ISSN: 2346-3910 en línea

revistageon@unillanos.edu.co

Universidad de los Llanos

Colombia

Céspedes Vargas, María Camila; Rodríguez Rodríguez,

Paula Andrea; Moreno Franco, Olga Lucia.

Nivel de implementación de las NIIF en las

funerarias de Villavicencio - Colombia

Revista GEON, Vol. 5, No. 1, 2018

Pág. 42-53

Disponible en: https://doi.org/10.22579/23463910.12

Esta publicación se encuentra bajo licencia: Creative Commons

ReconocimientoNoComercialSinObraDerivada 4.0 Internacional

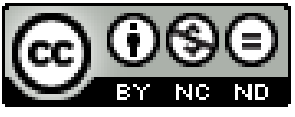

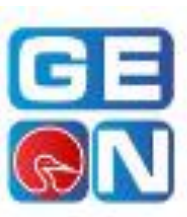

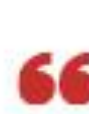

\section{Existe una percepción negativa en cuanto a la posibilidad que trae la adopción de las NIIF en adquisición de inversión} extranjera

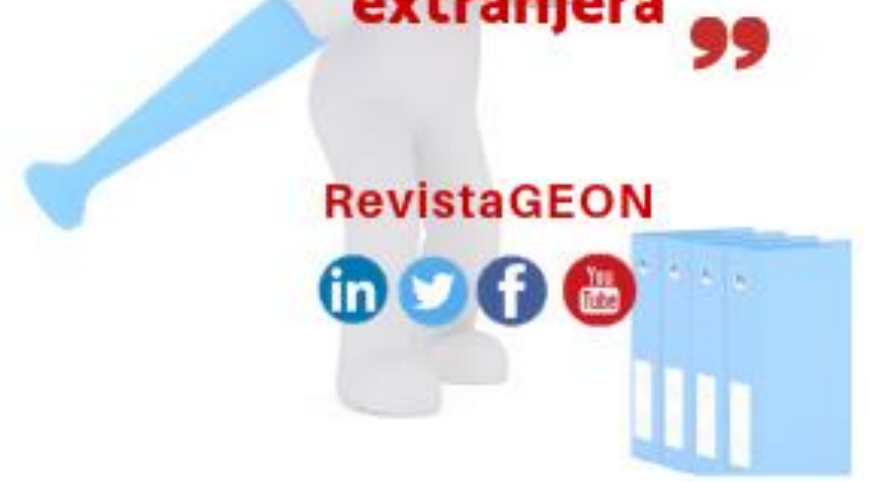

Céspedes Vorqas, M., Rodríquez Rodríquez, P., Franco, O. L, \& Tobar Martínez, I [2018]. Nivel de implementación de las NIIF en las funerarias de Villavicencio 


\section{REVISTA GEON}
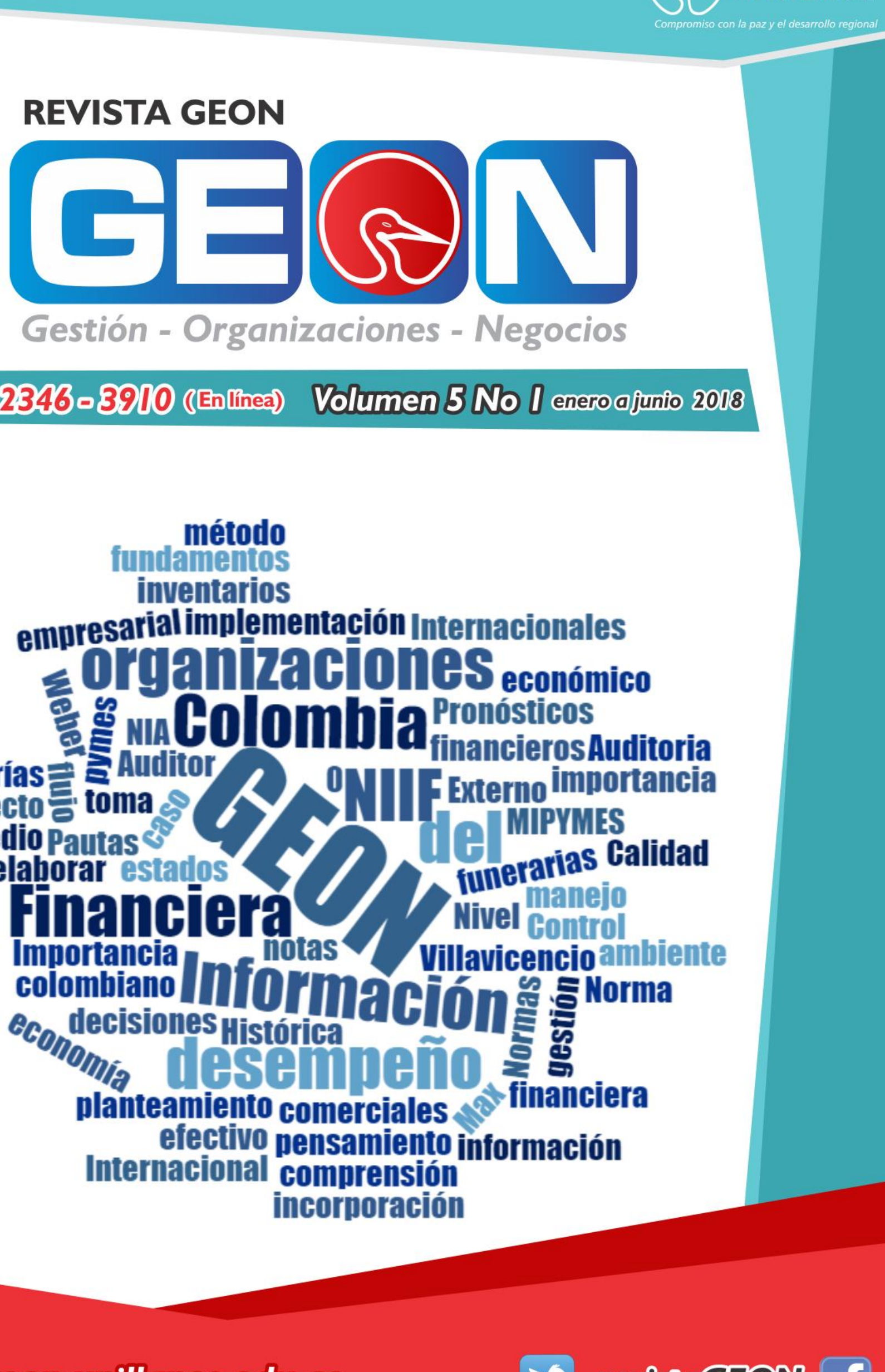

Gestión - Organizaciones - Negocios

ISSN 2346-3910 (Enlinea) Volumen 5No I enero a junio 2018

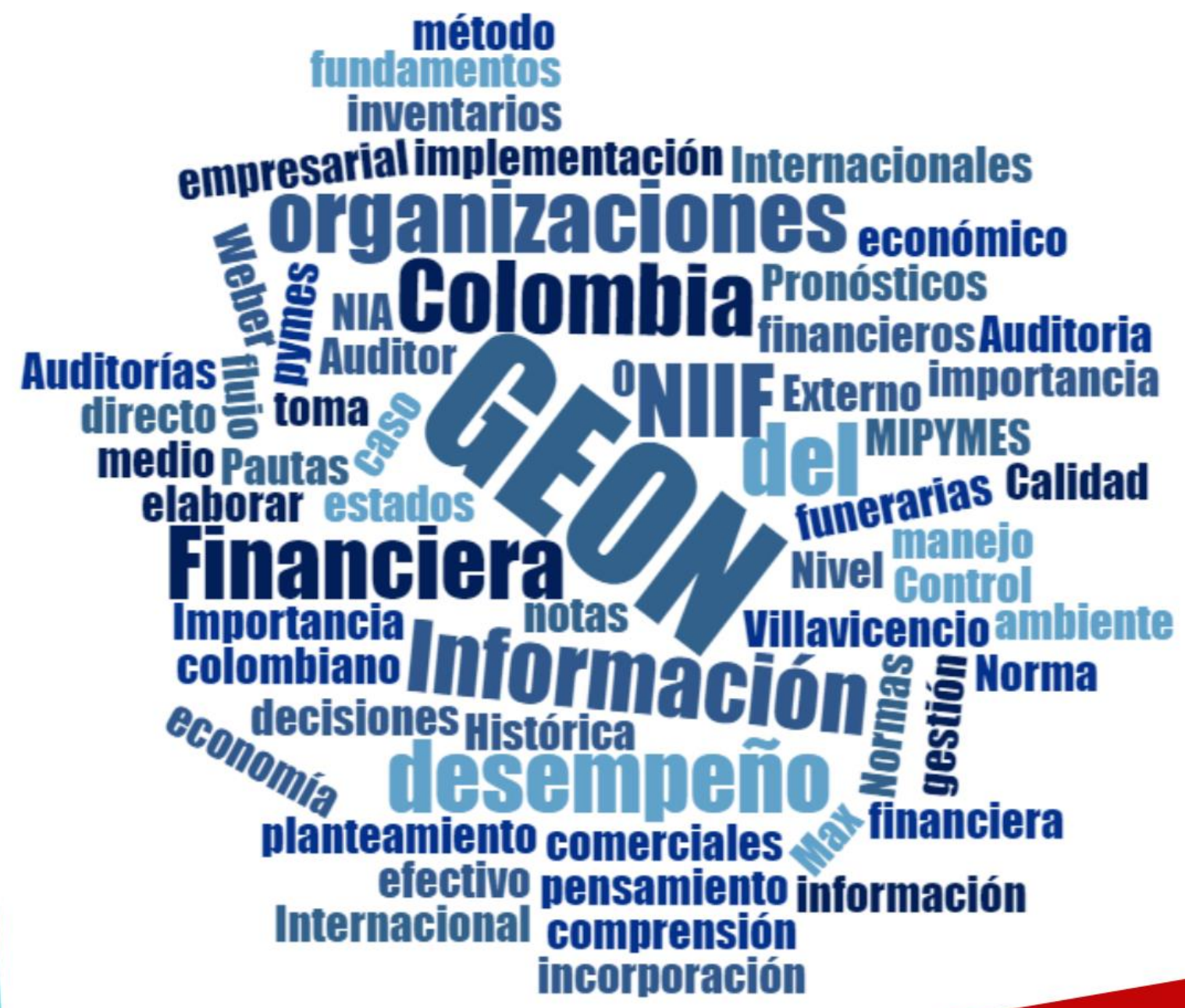

http://revistageon.unillanos.edu.co

revistaGEON f 


\title{
Nivel de implementación de las NIIF en las funerarias de Villavicencio - Colombia
}

\section{Level of implementation of the IFRS in the funeral homes of Villavicencio - Colombia}

María Camila Céspedes Vargas, Contador Público, Universidad Cooperativa de Colombia sede Villavicencio, camila1478@hotmail.com, Colombia

Paula Andrea Rodríguez Rodríguez, Contador Público, Universidad Cooperativa de Colombia sede Villavicencio, paulaandrearodriguezrodriguez@hotmail.com, Colombia

Olga Lucia Moreno Franco, Contador Público, Universidad Cooperativa de Colombia sede Villavicencio, lucymorenofranco@hotmail.com, Colombia

Julio Alexander Tobar Martínez, Especialista en revisoría fiscal y control de gestión, Universidad Cooperativa de Colombia sede Villavicencio, julio.tobar@campusucc.edu.co Colombia

\section{Resumen}

El presente artículo identifica y analiza el nivel de implementación de las normas internacionales de información financiera NIIF en PYMES funerarias de la ciudad de Villavicencio. Para alcanzar este propósito se desarrolló un Análisis Sistemático de Literatura por medio de un estudio de enfoque cuali-cuantitativo, apoyado en la investigación de tipo descriptiva, tomado como población a 6 funerarias, pymes, aplicando el instrumento de recolección de información al encargado del área contable de la empresa. Los hallazgos permiten evidenciar el bajo grado de cumplimiento de la normatividad en materia de adopción de las NIIF por parte de las funerarias de Villavicencio, pese a que existe una buena percepción generalizada de la norma internacional; Resultados con los cuales se formularon recomendaciones que viabilicen la implementación de las NIFF en la población objeto de estudio.

Palabras clave: NIIF, implementación, Pymes, percepción.

\begin{abstract}
This article identifies and analyzes the level of implementation of international financial reporting standards IFRS in funeral SMEs of the city of Villavicencio. To achieve this purpose, a Systematic Literature Analysis was developed through a qualitative-quantitative approach study,
\end{abstract}


supported by descriptive research, taken as a population to 6 funeral centers, SMEs, applying the information collection instrument to the area manager. accounting of the company. The findings allow to demonstrate the low degree of compliance with the regulations on the adoption of IFRS by funeral homes Villavicencio, although there is a good general perception of the international standard; Results with which recommendations were formulated that make possible the implementation of the NIFF in the population under study.

Keywords: IFRS, implementation, SMEs, perception.

\section{Introducción}

Las empresas a nivel global sin importar su tamaño, si son públicas o privadas, si son nacionales $\mathrm{o}$ multinacionales y sus directivos, administradores, gerentes, asesores, etc., deben obligatoriamente desarrollar la capacidad para adaptarse al cambio y mejoramiento continuo en todos los ámbitos, financiero económico etc., es decir, introducirse en el ambiente empresarial moderno (Oyola y Padilla, 2012; Puerto, 2010).

Bajo estos lineamientos las empresas del sector funerario a nivel nacional $\mathrm{y}$, particularmente, en la ciudad de Villavicencio deben aplicar las Normas NIIF como un paso necesario hacia la modernización administrativa - financiera, para la participación empresarial en un mundo cada vez más globalizado.

En este orden de ideas, las empresas presentan situaciones complejas de orden financiero las cuales requieren un tratamiento adecuado, por la exigencia del Gobierno Colombiano de adoptar las Normas Internacionales de Información Financiera en el país (a través de la Ley 1314 de 2009 y sus decretos reglamentarios), las cuales buscan dotar de una herramienta de transparencia y calidad a las empresas para afrontar el reto de competir en un mercado globalizado y financiero.

La aplicación de las NIIF representa un tema de aplicación profesional en el área financiera de las empresas, por lo cual los Contadores Públicos, como profesionales responsables de esta área empresarial, están obligados a tener un conocimiento amplio y un dominio efectivo de las mismas, puesto que las NIIF principalmente son herramientas de calidad y trasparencia de la información financiera antes que un tema de requisitos legales (Jánica, 2012).

Además hay que considerar que la norma exige que las pequeñas y medianas empresas del país a primero de enero de 2016 hallan empezado a aplicar las NIIF; sin embargo es de considerar que este plazo ya sea pospuesto por parte del estado ante la imposibilidad que las organizaciones cumplan con el plazo, puesto que la adopción de la norma exige cierto grado de estudio y de conocimiento, aunque estos cambios son necesarios para las pequeñas y medianas empresas, por lo que deben realizar la inversión necesaria 
en la implementación de las NIIF para PYMES.

Por lo anterior se adelantó una investigación de enfoque cualicuantitativo, y de tipo descriptiva, con el fin de identificar el nivel de implementación de las NIIF en las funerarias de Villavicencio, con el ánimo de formular recomendaciones que viabilicen la implementación de esta norma, para que estas empresas no sean sancionadas y se pueda adoptar de una vez por todas las NIIF, por lo menos en este sector empresarial de Villavicencio.

\section{Contexto teórico}

Para iniciar es preciso conceptuar lo que son las Normas Internacionales de Información Financiera "NIIF", para ello se acude a la definición contenida en el Reglamento General de la Contabilidad Comentarios Decreto 2649 de 1993, impreso por Legis (2012), en donde dice que:

"las NIIF corresponden a un conjunto único de normas legalmente exigibles y globalmente aceptadas, comprensibles y de alta calidad basados en principios claramente articulados que requieren que los estados financieros contengan información comparable, transparente y de alta calidad, que ayude a los inversionistas, $\mathrm{y}$ a otros usuarios, a tomar decisiones económicas. Las NIIF están diseñadas para que un país deje claramente las bases contables, los tributos y el impuesto sobre las ganancias y los valores patrimoniales por las cuales debe declarar sus activos y pasivos". (p. 74).
Aunque en la anterior definición no son tan explícitas algunas características destacada por Luna y Muñoz (2011), quienes exponen que las NIIF determinan los requerimientos para reconocer, medir, presentar y revelar la información financiera que es importante en los estados financieros de propósito general, los cuales satisfacen las necesidades de usuarios como accionistas, empleados, acreedores y el público en general. Además, el punto de referencia de las NIIF es el marco conceptual que se basa en los rasgos y nociones de la información que debe reflejarse en los estados financieros.

Se tratarán las NIIF como la parte fundamental de las normas que se deben cumplir y de las exigencias de estas, ya que los mercados actuales se encuentran en un "crecimiento económico del país, el cual está consolidando en su política comercial y de inversión mediante acuerdos comerciales que permitan generar mejores oportunidades laborales a los colombianos y el aumento del comercio y de la inversión nacional y extranjera" (Sosa. 2015).

Además, es apropiado tener en cuenta cuales serían los impactos que traerían para las entidades las NIIF, teniendo en cuenta en primera instancia que dicha adopción es un proceso complejo que afectaría los aspectos contables y no contables de cada una de las compañías. Este nuevo lenguaje de los negocios puede requerir cambios para el desarrollo de estos a través de la modificación en la ejecución de las transacciones (Acero y Cárdenas, 2011; Mantilla, 2012). 
En el plano nacional, la adopción de las NIIF es un tema que ha sido tratado desde hace varios años, pero que no se ha logrado materializar, pues han ocurrido varias discrepancias relacionadas con el abrupto cambio y la responsabilidad que debe asumir el Estado y las diferentes instituciones de la rama contable y económica, lo cual ha derivado en su aplazamiento, ya que implica modificar la reglamentación propia por las que proponen los expertos internacionales (Cárdenas y Manosalva, 2014).

Tabla 6. Cronograma de aplicación de las NIIF en Colombia

\begin{tabular}{ll}
\hline $\begin{array}{l}\text { Fecha de expedición de las } \\
\text { normas NIIF }\end{array}$ & $\begin{array}{l}\text { GRUPO 1 } \\
\text { diciembre de 2012 }\end{array}$ \\
\hline $\begin{array}{l}\text { Periodo de preparación } \\
\text { obligatoria }\end{array}$ & Año 2013 \\
\hline $\begin{array}{l}\text { Fecha de transacción-Balance } \\
\text { de apertura }\end{array}$ & 31 de diciembre de 2014 \\
\hline $\begin{array}{l}\text { Fecha de reporte-Estados } \\
\text { financieros NIIF }\end{array}$ & 31 de diciembre de 2015 \\
\hline
\end{tabular}

Fuente: Presidencia de la República, el Oficio 1200099881.

De acuerdo a la Ley 1314 de 2009, los estándares de contabilidad e información financiera deben ser aplicados por toda persona natural $\mathrm{o}$ jurídica que la normatividad vigente obligue a llevar contabilidad, al igual que los contadores públicos, funcionarios $\mathrm{y}$ demás personas delegadas para la preparación de estados financieros y otra información financiera, de su promulgación y aseguramiento, por lo tanto el proceso de convergencia a las NIIF aplica para todo aquel obligado a llevar contabilidad, y será exigido acorde al cronograma expuesto en la tabla 1 , en los grupos descritos de la tabla 2.
De otro lado hay que apuntar que se han realizado algunos estudios sobre las NIIF que ambienta el desarrollo de la presente investigación, entre ello se destacan:

El realizado por Lucumí y Pérez (2010), quienes abordaron la problemática que se presentó en el proceso de adopción de normas internacionales por parte del grupo empresarial Bavaria S.A., utilizando para su análisis una metodología descriptiva explicativa porque presentaba una situación actual, con sus variables y las analiza. Dicha investigación se basó en fuentes primarias como fue el personal del

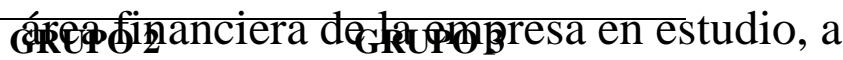

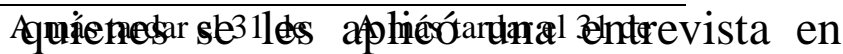

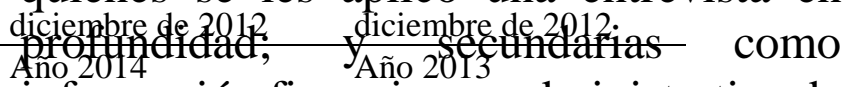
información financiera y administrativa de

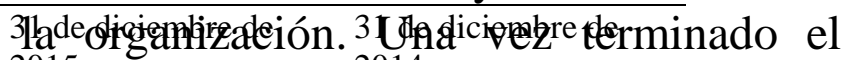
2015 ludiq los 2014 prdingipales resultados zmuestran que la empresa Bavaria S.A., se ajusta al cumplimiento de las normas internacionales de información financiera de acuerdo con lo dispuesto por los objetivos de la casa matriz.

Por su parte Andrade y Soto (2009), en su estudio abordaron el problema que debe enfrentar la empresa Aerovías DAP S.A con la incorporación del Plan de Convergencia NIC-NIIF, la cual sufrirá un fuerte impacto al enfrentar los nuevos desafíos que traería este moderno plan el cual provocaría un cambio cultural en los distintos niveles de la gerencia. Este estudio tuvo un alcance exploratorio y descriptivo; Los hallazgos encontrados en este estudio se establecieron al analizar el proceso de convergencia en donde se determinó que Aerovías DAP S.A va a tener un fuerte impacto al aplicar normas 
internacionales puesto que existirán tratamientos contables distintos para ciertas partidas del Balance General y el Estado de Resultados, dicho impacto no se pudo conocer en términos cuantitativos debido a que la empresa no facilito la información necesaria para calcularlo.

Tabla 7. Grupos de aplicación de la NIIF en Colombia

\begin{tabular}{|c|c|c|}
\hline GRUPO 1 & GRUPO 2 & GRUPO 3 \\
\hline $\begin{array}{l}\text { a) Emisores de valores; } \\
\text { b) Entidades de interés público; } \\
\text { c) Entidades que tengan activos totales } \\
\text { superiores a treinta mil (30.000) } \\
\text { SMMLV o planta de personal superior a } \\
\text { doscientos (200) trabajadores; que no } \\
\text { sean emisores de valores ni entidades de } \\
\text { interés público y que cumplan además } \\
\text { cualquiera de los siguientes requisitos: } \\
\text { i. Ser subordinada o sucursal de una } \\
\text { compañía extranjera que aplique NIIF; } \\
\text { ii. Ser subordinada o matriz de una } \\
\text { compañía nacional que deba aplicar } \\
\text { NIIF; } \\
\text { iii. Realizar importaciones (pagos al } \\
\text { exterior, si se trata de una empresa de } \\
\text { servicios) o exportaciones (ingresos del } \\
\text { exterior, si se trata de una empresa de } \\
\text { servicios) que representen más del } 50 \% \\
\text { de las compras (gastos y costos, si se } \\
\text { trata de una empresa de servicios) o de } \\
\text { las ventas (ingresos, si se trata de una } \\
\text { compañía de servicios), } \\
\text { respectivamente, del año } \\
\text { inmediatamente anterior al ejercicio } \\
\text { sobre el que se informa, o } \\
\text { iv. Ser matriz, asociada o negocio } \\
\text { conjunto de una o más entidades }\end{array}$ & $\begin{array}{l}\text { a) Empresas que no cumplan con los } \\
\text { requisitos del literal c) del grupo 1; } \\
\text { b) Empresas que tengan Activos } \\
\text { totales por valor entre quinientos } \\
\text { (500) y treinta mil (30.000) SMMLV } \\
\text { o planta de personal entre once (11) y } \\
\text { doscientos (200) trabajadores, y que } \\
\text { no sean emisores de valores ni } \\
\text { entidades de interés público; y } \\
\text { c) Microempresas que tengan } \\
\text { Activos totales excluida la vivienda } \\
\text { por un valor máximo de quinientos } \\
\text { (500) SMMLV o Planta de personal } \\
\text { no superior a los diez (10) } \\
\text { trabajadores, y cuyos ingresos brutos } \\
\text { anuales sean iguales o superiores a } \\
\text { 6.000 SMMLV. Dichos ingresos } \\
\text { brutos son los ingresos } \\
\text { correspondientes al año } \\
\text { inmediatamente anterior al periodo } \\
\text { sobre el que se informa. Para la } \\
\text { clasificación de aquellas empresas } \\
\text { que presenten combinaciones de } \\
\text { parámetros de planta de personal y } \\
\text { activos totales diferentes a los } \\
\text { indicados, el factor determinante para } \\
\text { dicho efecto será el de activos } \\
\text { totales. }\end{array}$ & $\begin{array}{l}\text { (a) Personas naturales o } \\
\text { jurídicas que cumplan los } \\
\text { criterios establecidos en el } \\
\text { art. } 499 \text { del Estatuto } \\
\text { Tributario (ET) y normas } \\
\text { posteriores que lo } \\
\text { modifiquen. Para el } \\
\text { efecto, se tomará el } \\
\text { equivalente a UVT, en } \\
\text { salarios mínimos legales } \\
\text { vigentes. } \\
\text { (b) Microempresas que } \\
\text { tengan Activos totales } \\
\text { excluida la vivienda por } \\
\text { un valor máximo de } \\
\text { quinientos (500) SMMLV } \\
\text { o Planta de personal no } \\
\text { superior a los diez (10) } \\
\text { trabajadores que no } \\
\text { cumplan con los } \\
\text { requisitos para ser } \\
\text { incluidas en el grupo } 2 \text { ni } \\
\text { en el literal anterior. }\end{array}$ \\
\hline
\end{tabular}

Fuente: Presidencia de la República, el Oficio 12-00099881.

La encuesta que se hizo con el propósito de entender el grado de preparación que tenía el personal interno para responder al reto de adoptar las NIIF arrojó resultados no muy favorables ya que los encargados de la emisión de información de la empresa no poseen los conocimientos necesarios para aplicar las NIIF. Finalmente se pudo comprobar que la aplicación del Plan de Convergencia en Chile provocaría un cambio sustancial en la forma de valorizar los hechos contables que rodean la actividad de Aerovías DAP S.A. por lo cual se recomienda que las empresas deben estar preparándose para dichos cambios capacitando a los profesionales, pero no solo a los contadores sino a todas las personas que 
toman decisiones dentro de la organización.

Más recientemente Cárdenas y Manosalva (2014) adelantaron una investigación con el propósito fundamental de analizar el impacto en la adopción de las NIIF en las empresas del grupo 1 del municipio de Ocaña Norte de Santander. Para el logro de este proyecto, se definieron tres objetivos específicos: Elaborar un diagnóstico sobre la preparación de las empresas del grupo 1 para la adopción, identificar los efectos que se produjeron internamente en las empresas estudiadas derivados del nuevo modelo contable y financiero, y establecer las principales diferencias entre el decreto 2649 y NIIF, para determinar los principales impactos contables en los estados financieros. Para ello el estudio se hizo utilizando una investigación de tipo descriptivo, utilizando la encuesta como técnica para la recolección de información que una vez obtenida fue sometida a un proceso de tabulación y análisis. Los resultados obtenidos en ese estudio permitieron identificar que la mayoría de los directivos tienen conocimiento de que las empresas en Colombia se encuentran en el proceso de adopción de NIIF.

Además, se identificaron las áreas donde se considera que la empresa sufrirá cambios por dicha adopción y se conocieron los principales efectos producidos por la adopción de este sistema internacional. Así mismo, una vez revisada la bibliografía sobre NIIF y el decreto 2649/93 se establecieron las diferencias entre las dos normas, lo cual permitió conocer el impacto que tendrán los estados financieros.

\section{Materiales y Métodos}

El Análisis Sistemático de Literatura se desarrolló por medio de un estudio de enfoque cuali-cuantitativo, apoyado en la investigación de tipo descriptiva.

Como población objeto se consideró a las seis (06) funerarias, pymes, con sede en la ciudad de Villavicencio, aunque es de anotar que se diseñó y se aplicó mediante la técnica de la encuesta al encargado del área contable de la funeraria.

Igualmente fue importante la consulta en fuentes secundarias, para ello se acudió a bases de datos indexadas como Redalyc (Red de Revistas Científicas de América Latina y El Caribe, España y Portugal); y Scielo. Donde se consultó sobre el tema afín, de donde se elegirá el material documental idóneo que permita realizar la discusión amplia y suficiente del tema, que servirá para realizar el artículo resultante del Análisis Sistemático de Literatura.

\section{Resultados}

En Colombia se han emitido normas para su adopción en todas las empresas de los diferentes sectores, e incluso en septiembre de 2012, la Presidencia de la República, en coordinación con los ministerios de Hacienda y Crédito Público y ministerio de Comercio, Industria y Turismo, hicieron pública la decisión de aplazar un año el proceso de adopción de las NIIF en Colombia. Decisión tomada al tener en cuenta los reportes de algunas superintendencias, que informaron del alto porcentaje de organizaciones que no 
estarían listas para cumplir el cronograma que se estableció previamente (Martínez et al., 2015).

4.1. Cumplimiento de la normatividad en materia de adopción de las NIIF por parte de las funerarias de Villavicencio

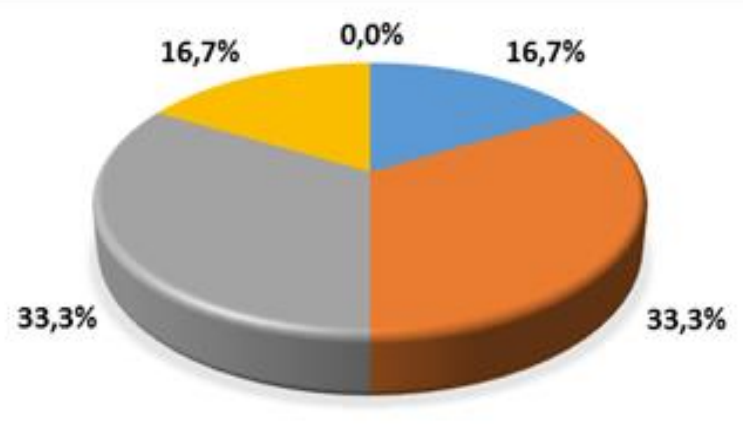

Mada a Masi nada algunas partes aCasi todo a Todo

Figura 1. Nivel de conocimiento de las NIIF en el área contable de la funeraria

Fuente: El estudio.

De acuerdo a la normatividad vigente, para el primer día del presente año se debió haber empezado con la aplicación de las NIIF en las pequeñas y medianas empresas colombianas (Legis, 2012), lo que demanda tener el conocimiento sobre la norma para su adecuada aplicación, sin embargo los resultados de la aplicación de la encuesta indican sólo el $16.7 \%$ del área contable de estas empresas tiene conocimiento de casi todas las NIIF; el $33.3 \%$ manifiestan conocer algunas y el $50 \%$ restante tienen casi nada o nada de conocimientos (Ver figura 1), lo cual revela un conocimiento limitado de la norma, lo que de acuerdo a Salazar (2013) impide la adecuada adopción de las NIIF en las empresas, pues es fundamental en primera medida conocerlas para luego implementarlas.

En este sentido Ruiz (2014) apunta que el conocimiento de la norma es un elemento fundamental para realizar la adecuada implementación, aunque es de anotar que el desconocimiento de la norma en Colombia puede ser causal de un delito por omisión, por lo menos así lo manifiesta Borrero (2014) en su ensayo de grado, cuyo tema es el impacto de las NIIF en las pequeñas empresas colombianas.

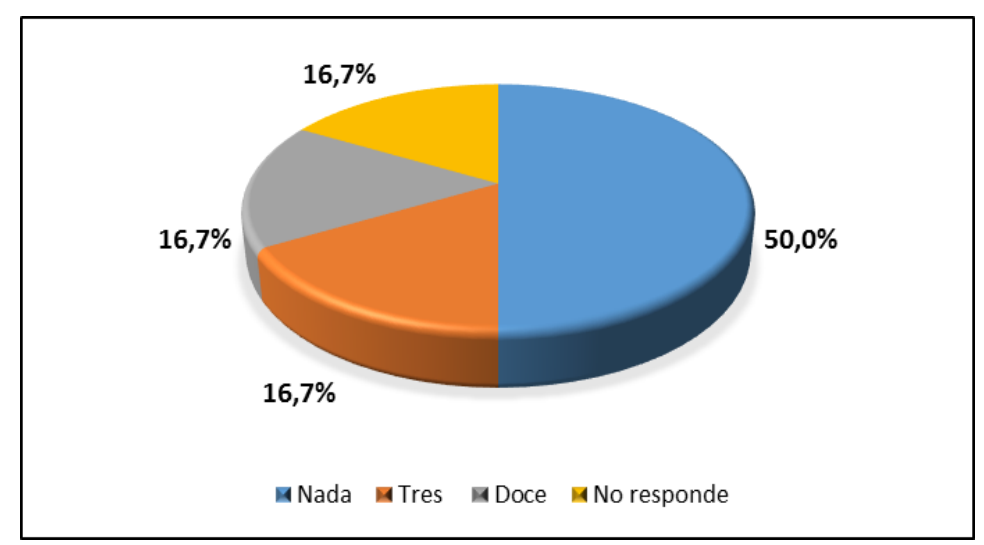

Figura 2. Tiempo de aplicación de las NIIF en la funeraria

Fuente: El estudio.

Por su parte, al indagar sobre el tiempo que en la Pyme empezó a aplicar las NIIF se encontró que el $16.7 \%$ de ellas lo han venido haciendo hace 12 meses; por su parte la mitad las funerarias de Villavicencio admiten no haber empezado a aplicar las NIIF y otro $16.7 \%$ dicen sólo llevar 3 meses (figura 2), con lo cual se exponen a las sanciones prevista por la legislación colombiana, pues el calendario establece que a primero de enero de este año se debió haber hecho el balance inicial bajo NIIF y continuar con su aplicación (Legis, 2012). 


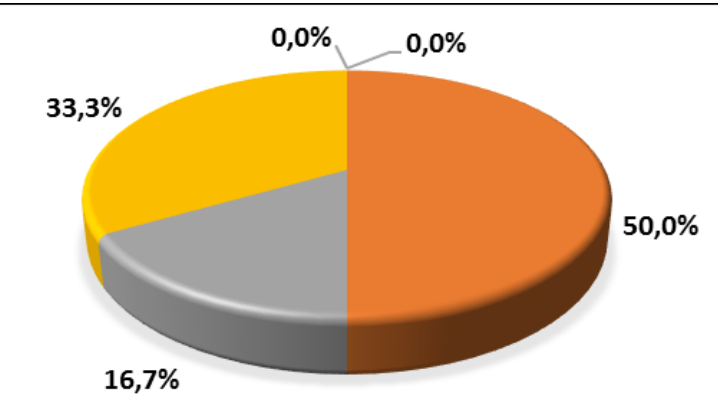

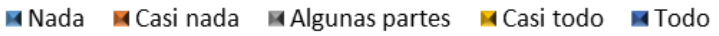

Figura 3. Nivel de implementación de las NIIF en la funeraria

Fuente: El estudio.

Hoy en día las empresas del grupo 2, entre ellas las funerarias Pymes de Villavicencio, deberían de haber realizado los cambios pertinentes para la implementación de las NIIF, pero como se evidencia en la figura 3 sólo el $33.3 \%$ de estas empresas han implementado casi todas las NIIF, lo que se da en razón a que ya cuentan con el balance de inicio del ejercicio contable anual; el $16.7 \%$ tienen algunas partes pero aún no han iniciado el primer año de ejercicio contable aplicando la norma internacional y el $50 \%$ restante tienen casi nada, lo que se traduce que no van a cumplir con los requerimientos de ley en cuanto al plazo dado por el Gobierno Nacional, exponiéndose a sanciones.

Estos resultados indican que sólo el $33.3 \%$ de las Funerarias Pymes de Villavicencio han cumplido con la normatividad que regula el estado colombiano en materia de adopción de las NIIF para Pymes, pues se encuentran adelantando su primer año fiscal en la adopción, aunque es preocupante que la mitad de las empresas tengan casi nada implementado, por lo que no están al día con el calendario exigido por el Gobierno Nacional, sin embargo esta es una realidad que ya ha sido expuesta por autores como Martínez et al. (2015) y Cárdenas y Manosalva (2014), quienes se han pronunciado sobre lo lento que ha sido el proceso de actualización de la normatividad contable en Colombia.

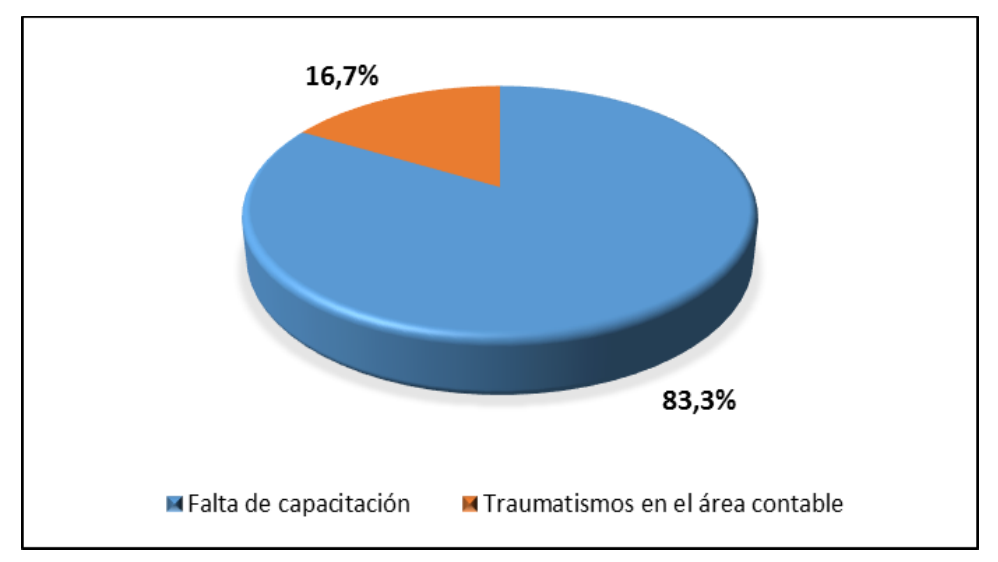

Figura 4. Dificultades para implementar las NIIF Fuente: El estudio.

De otro lado, hay que apuntar que en todas las funerarias se manifiestan dificultades para implementar las NIIF, siendo estas en su totalidad la falta de capacitación (83.35) y la generación de traumatismos en el área contable (16.7\%) son las principales dificultades para su implementación. En materia de capacitación, autores como Molina (2013) y Gutiérrez (2008) han destacado la importancia que tiene la preparación técnica del contador público para la implementación de la NIIF, por ello proponen que se adelanten programas de formación técnica con el contenido académico amplio y suficiente para poder dar respuesta a las exigencias de la adopción de la norma internacional. 


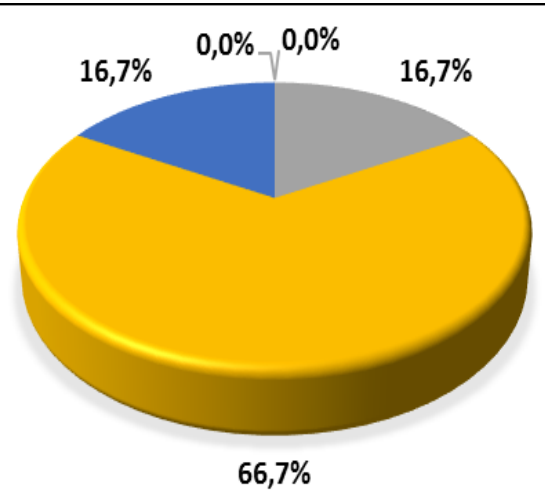

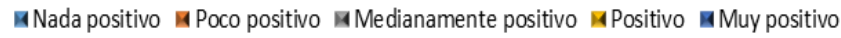

Figura 5. Nivel de percepción positiva que posee el área contable de la funeraria para implementar las NIIF

Fuente: El estudio.

Pese a los anteriores resultados en el $83.4 \%$ de las áreas contable de las funerarias de Villavicencio consideran como positivo o muy positivo la adopción de las NIIF en estas Pymes, lo que evidencia que a pesar de los niveles de implementación, las dificultades que han tenido para su adopción y los plazos otorgados por el Gobierno Nacional se mantiene una actitud positiva, lo que contribuye a llegar con mayor prontitud a materializarse en los procesos contables de las funerarias de Villavicencio.

Es de anotar que el $16.7 \%$ de los encuestados perciben medianamente positiva esta implementación, pues tal vez conocen argumentos como el de Londoño (2015), quien sostiene que al aplicar las NIIF las organizaciones pueden ver afectados sus indicadores de forma significativa por utilizar activos que se encuentran totalmente depreciados en libros, igualmente porque a mayor valor comercial de la propiedad Planta y equipo existirá más alto el impacto negativo en los indicadores financieros de rentabilidad, puesto que el nivel de activos involucrados aumentará la rentabilidad esperada por los socios y accionistas. O el de Salas (2015), quien advierte sobre la importancia de definir la política contable en materia de cartera, por cuanto las tasas de interés y los tiempos de cobro, son vistos como pieza clave para la liquidación de la deuda conforme al estándar internacional.

\subsection{Nivel de percepción de la} contribución de las NIIF para que inversionistas, nacionales y extranjeros, se interesen en invertir en las funerarias de Villavicencio

La encuesta aplicada también contó con preguntas para identificar el nivel de percepción, de quienes manejan el área contable, sobre la contribución de las NIIF para que inversionistas, nacionales $\mathrm{y}$ extranjeros, se interesen en invertir en estas Pymes villavicenses, encontrando que:

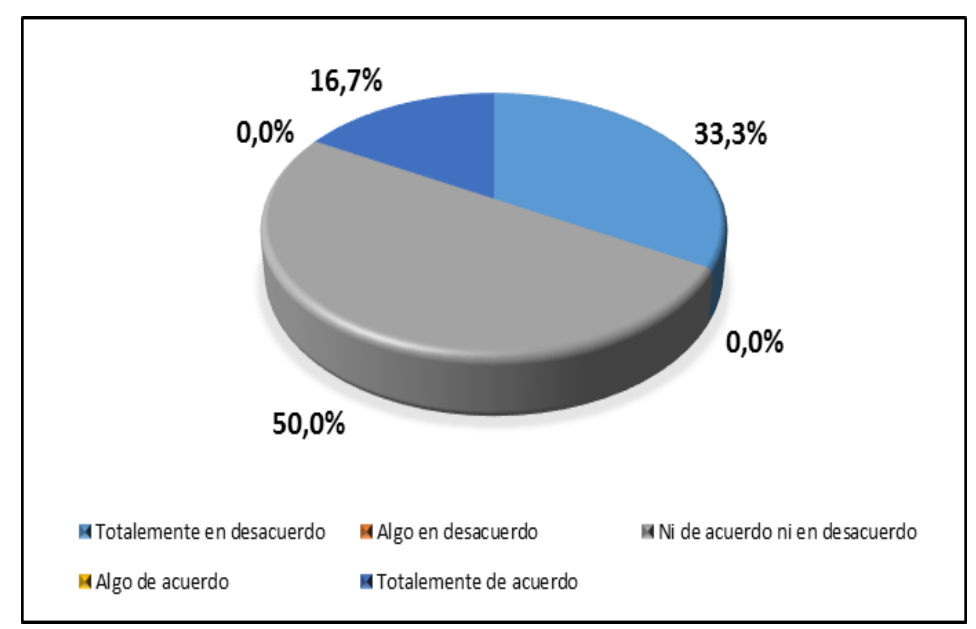

Figura 6. Grado de acuerdo en contribución de las NIIF en la atracción de inversionista a la funeraria Fuente: El estudio.

Los resultados plasmados en la figura 6 evidencian que existe en gran medida un grado de incertidumbre tendiente a desacuerdo, como uno de los grandes 
beneficios que concede las NIIF, el de ampliación de las opciones de inversión extranjera (Legis, 2012), pues en el 50\% de las funerarias de Villavicencio no están de acuerdo ni en desacuerdo con la afirmación que la implementación de las NIIF en la funeraria ha contribuido para que los inversionistas (nacionales y extranjeros) se interesen en invertir la organización, y el $33.3 \%$ está en desacuerdo; además la encuesta encontró que tampoco la ven como una oportunidad a futuro, lo que va en contravía del pensamiento de Cano (2010), quien estima que "cuando las normas son bien conocidas y se aplican de manera uniforme, los inversionistas pueden interpretar correctamente la situación financiera de la firma" (p. 210), lo que debería ser un factor atrayente para la inversión extrajera, pues de acuerdo a Muñoz et al. (2012) "la presencia de multinacionales es positiva para el desarrollo de las pymes y, por consiguiente, para el desarrollo económico del país" (p. 226).

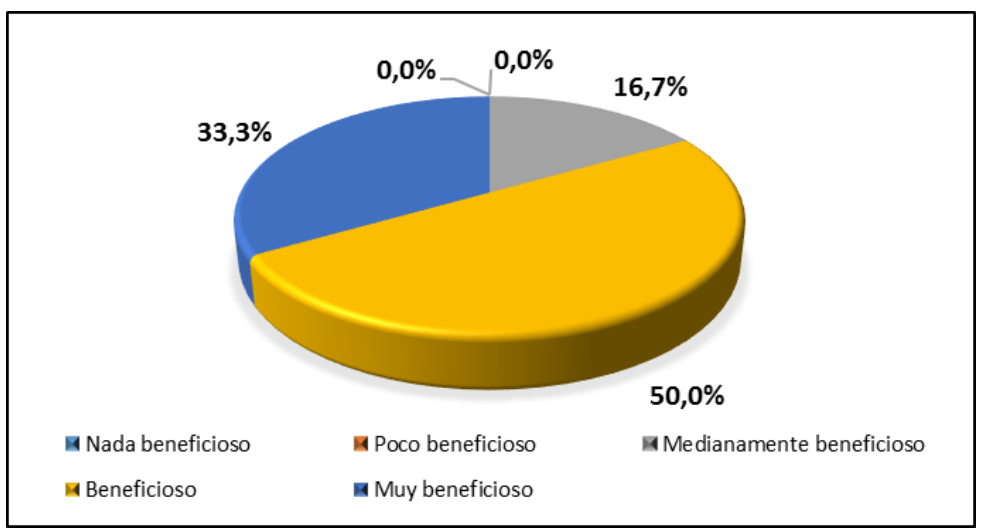

Figura 7. Percepción del beneficio de las NIIF para el desarrollo de relaciones comerciales internacionales con aliados, proveedores y/o clientes Fuente: El estudio.

No obstante, en la figura 7 se puede identificar que la postura optimista de las pymes objeto de estudio en cuanto a los beneficios de las NIIF en el desarrollo de relaciones comerciales internacionales con aliados, proveedores y/o clientes, pues estiman que la adopción es beneficiosa o muy beneficiosa para las funerarias de Villavicencio, ya que permitirá abrir nuevas relaciones con posibles aliados, o buscar proveedores que brinden mejores garantías, hasta captar nuevos clientes en el plano internacional.

En consideración a todo lo anterior la totalidad de las funerarias villavicenses $(100 \%)$ ven la adopción de las NIIF como una oportunidad para el fortalecimiento financiero de la empresa, por lo que se encuentran corrigiendo sus falencias para poder adoptarlas adecuadamente.

\subsection{Recomendaciones que} viabilicen la implementación de las NIIF en las funerarias de Villavicencio

Los resultados evidencian las falencias existentes en la adopción de las NIIF por parte de las funerarias villavicenses, es por ello por lo que se recomienda adelantar las siguientes acciones:

El contador público debe asumir su papel de garante de los procedimientos contables de la empresa para la cual se despeña, por lo tanto es preciso que mantenga actualizado en temas relacionados a su quehacer profesional, así estos demanden la inversión de recursos propios.

Además, hay que reconocer que el profesional de la contaduría no puede ejercer su adecuado papel en la implementación de las NIIF si la gerencia de la empresa no destina los recursos 
suficientes para tal fin, por ello es preciso que las funerarias de Villavicencio no solamente se interesen por cumplir con la normatividad, sino que apropien tales recursos, tomándolos como una inversión que podrá ser recuperada en un futuro.

También es recomendable dar a conocer a las Instituciones de Educación Superior sobre la necesidad urgente de desarrollar programas que permitan hacerles seguimiento y acompañamiento a sus profesionales egresados, donde puedan establecer y determinar el grado de preparación, actualización profesional en que se encuentran y en qué condiciones están ejerciendo su quehacer profesional que permitan proyectar una buena imagen de su alma mater, por medio de la actualización en temas contables actuales, como la implementación de las NIIF.

\section{Conclusiones}

Pese a que a la fecha, de acuerdo con el calendario contable y la normatividad colombiana, ya se debería estar acabando el primer año contable de implementación de las NIIF en las pymes domiciliadas en el territorio nacional, la mayoría de las funerarias de la ciudad de Villavicencio aún no han avanzado lo suficiente para estar a este nivel, por lo cual se exponen a las sanciones del caso.

Así mismo, se pudo identificar que existe una percepción negativa en cuanto a la posibilidad que trae la adopción de las NIIF en adquisición de inversión extranjera, sin embargo la mayoría de las funerarias villavicenses aun ven con buenos ojos la contribución de las normas internacionales en entablar nuevas relaciones con aliados, proveedores $\mathrm{y}$ clientes extranjeros.

En consideración se formularon recomendaciones tendientes a viabilizar la implementación de las NIIF en las pymes objeto de estudio, siendo la constante la capacitación de contador público y la disponibilidad de recursos por parte de la gerencia.

\section{Referencias bibliográficas}

Acero, A. J. y Cárdenas, C. (2011). La revisoría fiscal frente a las Normas Internacionales de Información Financiera. Ensayo de grado. Bogotá: Universidad Militar Nueva Granada.

Andrade, N. y Soto, R. (2009). Plan de Convergencia a Normas Internacionales de Información Financiera. Trabajo de grado. Chile; Universidad de Chile. 128 p.

Borrero, X. M. (2014). Evolución de las NIIF para pymes y su impacto en las pequeñas empresas en Colombia. Ensayo de grado. Bogotá: Universidad Militar Nueva Granada.

Cárdenas, D.F. y Manosalva, I.Y. (2014). Impacto en la adopción de las NIIF en las empresas del grupo 1 del municipio de Ocaña Norte de Santander. Trabajo de grado. Ocaña: Universidad Francisco de Paula Santander. 98 p.

Cano, A. M. (2010). Algunas experiencias exitosas en Latinoamérica relacionadas con la estandarización contable. Revista Facultad de Ciencias Económicas: Investigación $\quad y$ Reflexión, 18(2), pp. 207-226.

Gutiérrez, C. (2008). Propuesta de conocimientos y competencias requeridas por el profesional contable en Chile derivado de 
la adopción de las NIIF. CAPIC REVIEW, (6), pp. 47-60.

Jánica, F. (2012). Las NIIF y su impacto en el Contador Colombiano. [Artículo en línea]. Colombia: Instituto Nacional de Contadores Públicos. Recuperado de: http://incp.org.co/Site/2012/agenda/5-ni.pdf

Legis (2012). Reglamento General de la Contabilidad - Comentarios Decreto 2649 de 1993. Bogotá: Editorial Legis.

Londoño, L. A. (2015). Impacto financiero en la aplicación de las normas Internacionales de Información Financiera de valoración y registro de la propiedad planta y equipo en los indicadores financieros de las Pymes en Colombia. Trabajo de grado. Medellín: Universidad EAFIT.

Lucumí, M. y Pérez , V. (2010). Adopción de las Normas Internacionales de Información Financiera. Trabajo de grado. Santiago de Cali: Universidad ICESI. 122 p.

Luna, J. y Muñoz L. J. (2011). Colombia: Hacia la adopción y aplicación de las NIIF y su importancia. Revista Adversia, Universidad de Antioquia, enero-junio, pp. 26-43.

Martínez, Y. P.; León, M. A.; Santamaría, J. A. y Vargas, I. M. (2015). NIIF aplicadas a los estados financieros básicos y consolidados. Bogotá: Institución Universitaria Politécnico Grancolombiano. $56 \mathrm{p}$.

Molina, R. (2013). NIIF para las PYMES: ¿La solución al problema para la aplicación de la normativa internacional? Contabilidad y Negocios, 8 (16), pp. 21-34.
Muñoz, Alberto et al. (2012). Evaluación del impacto de las multinacionales en las pymes de la ciudad de Barranquilla: Realidad de las empresas vinculadas vs. la percepción de las empresas no vinculadas. Pensam. gest., (32); pp. 225247.

Oyola, M. A. y Padilla, L. M. (2012). El reto frente a la globalización: la competitividad desde un enfoque sistémico. Gestión \& Desarrollo, 9 (1), pp. 161-173.

Puerto, D. P. (2010). La globalización y el crecimiento empresarial a través de estrategias de internacionalización. Pensamiento y Gestión, (28), pp. 171-195.

Ruiz, D. (2014). Impacto de la aplicación de las NIIF en las empresas de Colombia. Bogotá: Universidad Militar Nueva Granada.

Salazar, E. E. (2013). Efectos de la implementación de la NIIF para las PYMES en una mediana empresa ubicada en la ciudad de Bogotá. Cuadernos de Contabilidad, 14 (35), pp. 395-414.

Sosa, J. M. (2015). Implicaciones del Decreto 0302 de febrero 20 de 2015 en la labor del Revisor Fiscal. Bogotá: Pontificia Universidad Javeriana. Recuperado de: http://docplayer.es/5878782-Educacioncontinua-y-consultorias-implicaciones-deldecreto-0302-de-febrero-20-de-2015-en-lalabor-del-revisor-fiscal.html

Villareal, K. L. (2014). Políticas contables aplicables para pymes NV Gestión y Gerencia SAS, Trabajo de grado inédito. Bogotá: Universidad Militar Nueva Granada. 